\title{
Optimal MTM Spectral Estimation Based Detection for Cognitive Radio in HDTV
}

\author{
Manjunath Kashyap Jataprolu*, Ravinder David Koilpillai ${ }^{\dagger}$ and Srikrishna Bhashyam ${ }^{\dagger}$ \\ *Bell Labs India Research, Bangalore, India, (e-mail: manjunath_kashyap.jataprolu@alcatel-lucent.com) \\ ${ }^{\dagger}$ Department of Electrical Engineering, Indian Institute of Technology at Madras, India, (e-mail:\{koilpillai,skrishna\}@ee.iitm.ac.in)
}

\begin{abstract}
Cognitive radio based systems rely on spectrum sensing techniques to detect whitespaces to exploit. For the sake of ease of implementation, simple schemes such as energy detector have been proposed and studied widely. However, such simple schemes perform far from optimally, thereby affecting the performance of the underlying system. On the other hand sophisticated detectors are difficult to implement, giving rise to a trade-off. This paper explores the idea of using spectral estimates for detection. The case of HDTV based cognitive radio is explored and an optimal detection scheme following multi taper estimation is proposed and studied.
\end{abstract}

\section{INTRODUCTION}

Software Defined Radio (SDR) [1] has been proposed as a solution to the spectrum scarcity problem [2] in literature. Cognitive radio systems allow for unlicensed users (secondaries) to exploit vacancies in frequency bands to transmit without interfering with the licensed (primary) user's transmissions. Investigations by FCC have pointed towards using television bands for cognitive radio applications due to the large fraction of underutilized spectrum and recent efforts have focused on the development of a Wireless Regional Area Network (WRAN) standard along these lines (IEEE 802.22).

To avoid interfering with primaries, the cognitive radio needs to sense the radio spectrum. Traditionally methods such as energy sensing, etc. have been proposed as simple solutions for this problem. On the other hand cyclostationary feature detection, tone detection, waveform based sensing, etc. perform better at the cost of increased cost and complexity. In this work, we propose a detection algorithm that performs better, while being easy to implement and analyze, with the idea of estimate and detect. For the HDTV case, Thomson's Multi Taper Method has been suggested by Haykin [3] et al to be optimal in a certain sense. We look at estimating the power spectral density and using this to detect the signal.

This paper has been organized as follows. In Section II we give describe ATSC transmission scheme and the system considered. Section III, briefly reviews Thomson's multi taper method for spectral estimation. Section IV talks about the main contributions of this work. The idea of detection following spectral estimation is introduced and the problem is framed in terms of the Neyman-Pearson criterion. This is solved to obtain the optimal detector post spectral estimation. In Section V we study the performance of the proposed method using simulations. In conclusion, we summarize some of the improvements due to the proposed detector and the allied benefits.

\section{SYSTEM MODEL}

We consider a simple cognitive radio system with a single HDTV transmitter and secondary receiver. In this simplified IEEE 802.22 setup, we assume that there are no interferers (or are absorbed into the AWGN). The receiver has prior knowledge of the band of transmission from MAC layer information [4] and senses in this band.

The primary uses an 8-VSB modulation scheme [5]. Vestigial Sideband Modulation is a practical implementation of Single Sideband Modulation where the pass-band cut-off is gradual. The coded and interleaved data bits get mapped to a symbol stream $\left(s_{d}[n]\right)$ of 8 equiprobable levels. The data stream prior to modulation, and thereby the output symbol stream, are white.

$$
R_{s_{d} s_{d}}=E\left[s_{d}[i] s_{d}^{*}[j]\right]=\delta_{i j}
$$

In practice, a $2 X$ upsampled stream $(\{s[n]\})$, is pulse shaped with a finite-length SRRC filter $(\mathbf{h})$. After adding a constant pilot $(p)$, the baseband equivalent is then filtered to get the VSB spectrum which is upconverted to the designated RF and transmitted. The receiver downconverts the signal and extracts the baseband equivalent. We assume a single-tap Rayleigh fading $(\{\alpha[n]\})$ channel and AWGN $(\{w[n]\})$ at the receiver. The case with multiple taps can be handled similarly.

$$
x[n]=\alpha[n]\left(\mathbf{h}^{T} \mathbf{s}_{n}+p\right)+w[n]
$$

Even a filter length of 10 periods spans about 100 symbols owing to the high sampling rate of around $20 \mathrm{MHz}$. The shaped output process is given by $x_{T}[n]=\mathbf{h}^{T} \mathbf{s}_{n}$. Here textbf $s_{n}$ is the vector of symbols that contribute to the output at time $n$. This can be seen a weighted sum of i.i.d random variables. As $h$ is long, we can resort to extensions of the Central Limit Theorem (CLT) [6] to say that $x_{T}[n]$ is approximately Gaussian distributed and calculate its parameters as follows:

$$
\begin{gathered}
\text { Mean } \\
E\left\{x_{T}[n]\right\}=E\left\{\mathbf{h}^{T} \mathbf{s}_{n}\right\}=\mathbf{h}^{T} E\left\{\mathbf{s}_{n}\right\}=0 \\
\text { Variance } \\
\operatorname{Var}\left\{x_{T}[n]\right\}=E\left\{\mathbf{h}^{T} \mathbf{s}_{n} \mathbf{s}_{n}^{H} \mathbf{h}^{*}\right\}=\mathbf{h}_{d}^{*}=\sigma_{s}^{2} R_{h_{d} h_{d}}[0]
\end{gathered}
$$

where $\mathbf{h}_{d}$ is the filter downsampled twice. As $\mathbf{s}_{d, n}$ is white, the correlation matrix, $E\left\{\mathbf{s}_{d, n} \mathbf{s}_{d, n}^{H}\right\}$ is scaled unit matrix $\mathbf{I}$.

Using actual HDTV data ${ }^{1}$, we plot the distribution of $x_{T}[n]$ in Figure 1 . We see that the histogram looks very

\footnotetext{
${ }^{1}$ Observed HDTV (8-VSB) transmitted data obtained from the Indian Institute of Science (courtesy Dr. Chandra Murthy)
} 
much like a Gaussian. Further, a Jarque-Bera test [7] on the observed data admitted the Gaussian hypothesis with a confidence of $95 \%$ (usually considered sufficient). For

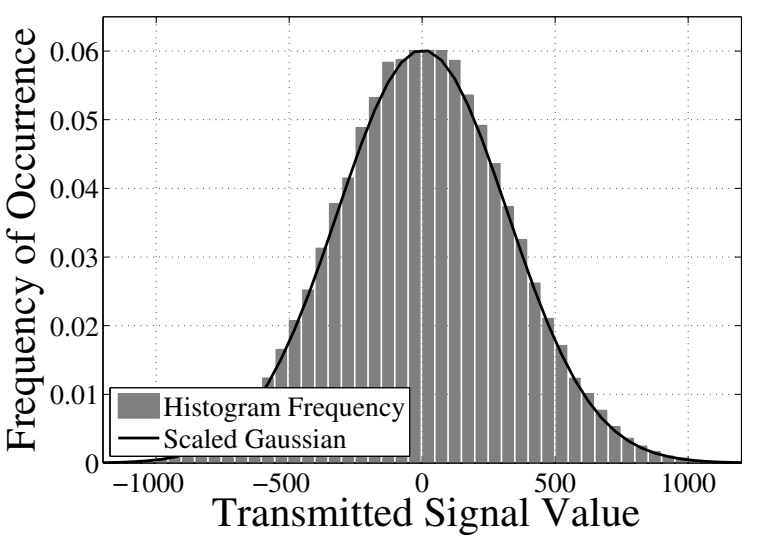

Figure 1: Histogram of Actual Transmitted Data - 100 bins

single-tap Rayleigh fading, $\alpha[n]$ is a zero-mean complex Gaussian with unit variance $\left(\sigma_{\alpha}^{2}\right)$. The noise variance is denoted by $\sigma_{w}^{2}$. The receiver is assumed to know the SNR, owing to ease and accuracy of its estimation due to slow variation in the HDTV SNR.

\section{Preliminaries: Multi Taper Method Based SPECTRAL ESTIMATION}

As the first of two steps, we look at the problem of spectral estimation which has been well studied in literature. In [3], Haykin proposed the use of Thomson's multi taper method [8] and showed it to be an optimal non-parametric estimation method for a hybrid spectrum such as that of HDTV containing continuous as well as line components. Motivated by this, we choose to employ the multi taper method.

A typical non-parametric estimator is the windowed periodogram. Varying the data window represents varying across the bias-variance trade-off. It is not possible to simultaneously decrease both the bias and variance of an estimator with the same data. Thomson's method [8] tries to find the optimal trade-off point. Also, as shown in [9], non-parametric methods do not handle line spectra well as they assume a continuous spectrum and do not resolve line spectra well. In [8], this concern is also addressed through the MTM.

MTM uses Discrete Prolate Spheroidal Wave functions (DPSW) which were introduced by Slepian as a solutions to the spectral concentration problem [10]. DPSW correspond to sequences of a given length $(N)$ with maximal spectral concentrations in a specified band (of width $W$ ). The DPSW are solutions of the Toeplitz matrix eigenvalue equation

$$
\mathbf{T}(N, W) \mathbf{v}_{k}(N, W)=\lambda_{k}(N, W) \mathbf{v}_{k}(N, W)
$$

where

$\mathbf{T}(N, W)_{m n}= \begin{cases}\frac{\sin [2 \pi W(m-n)]}{[\pi(m-n)]}, & m, n=0, \ldots, N-1 \text { and } m \neq n \\ 2 W & \text { for } m \neq n\end{cases}$

These sequences form an orthonormal set with decreasing eigenvalues correspond to increasing spectral leakage. MTM uses these windows to minimize spectral smearing due to windowing. Multiple windows are used to reduce variance of the estimation through averaging. The 'crude' version of the method [8] is as follows:

1) Apply the first $K$ DPSW sequences with some leakage bandwidth $W$ as windows to the $N$-point observed data.

$$
x_{k}[n]=x[n] . v_{k}[n](N, W)
$$

2) Compute the $N$ point periodograms of these windowed sequences

$$
\hat{S}_{k}\left(f_{i}\right)=\left|\sum_{n=0}^{N-1} x(n) v_{k}[n](N, W) e^{-j 2 \pi f_{i} n}\right|^{2}
$$

These eigenspectra represent spectral estimates with increasing leakage.

3) Crude MTM estimate is a linear combination of the eigenspectra with weights as scaled eigenvalues:

$$
\hat{S}_{\text {crude }}\left(f_{i}\right)=\sum_{k=0}^{K-1} a_{k} \hat{S}_{k}\left(f_{i}\right)
$$

where the weights $a_{k}$ are defined as $a_{k}=\frac{\lambda_{k}}{\sum_{i=0}^{K-1} \lambda_{i}}$ The bias-variance trade-off is now replaced by a three way bias-resolution-variance trade-off [3] with $C_{0}$ and $K$ as the tunable parameters. Setting $K=\lfloor 2 N W\rfloor$ has been shown to minimize the variance within the trade-off [3], owing to optimal averaging, and the problem is now of biasresolution trade-off. The optimal trade-off point is decided empirically while varying the value of $W$. It has been noted in [3] that a $K$ of $2-8$ gives the best estimates for a reasonable sample size.

Thomson in [8] introduces an improved set of weights $\left\{d_{k}(f)\right\}$ that are arrived at by minimizing certain meansquare error criteria. However, for the sake of simplicity in implementation and analysis, we restrict ourselves to the crude method. The proposed scheme would work well with the adaptive-weights algorithm also, albeit the analysis would be more involved.

\section{Detection Following Estimation}

Having dealt with estimation, we proceed to the second step of signal detection using spectral estimates. A brief literature survey indicates two attempts at this problem. The first one [11] makes use of the complete spectral estimate

$$
T_{\text {Chiang }}=(K-1) \sum_{i=0}^{N-1} \ln \left[\hat{S}_{M T M}\left(f_{i}\right)\right] \underset{\mathcal{H}_{0}}{\stackrel{\mathcal{H}_{1}}{\gtrless}} \xi_{\text {Chiang }}
$$


while the second [12] considers only the MTM estimate of the DC tone strength as a heuristic test-statistic

$$
T_{\text {Wang }}=\hat{S}_{M T M}\left(f_{0}\right) \underset{\mathcal{H}_{0}}{\stackrel{\mathcal{H}_{1}}{\gtrless}} \xi_{W a n g}
$$

Both the methods are shown to perform better than simple energy detector and cooperative detection schemes using the energy detector. However, both methods are suboptimal with reference to Neyman-Pearson.

For our system model explained in Section II we have an MTM estimates at $N$ frequencies. Redefining hypotheses in terms of these new "observations", we look to solve the detection problem stated below:

Neyman-Pearson criterion: We are given a set of estimates (observations) $\hat{S}_{M T M}^{o b s}\left(f_{i}\right),(i=0,1, \ldots, N-1)$, drawn as per the model

$$
\begin{array}{ll}
\mathcal{H}_{1}: & \hat{S}_{M T M}\left(f_{i}\right)=(\mathbf{x}+\mathbf{w})^{H} \boldsymbol{\Omega}\left(f_{i}, N\right)(\mathbf{x}+\mathbf{w}) \\
\mathcal{H}_{0}: & \hat{S}_{M T M}\left(f_{i}\right)=\mathbf{w}^{H} \boldsymbol{\Omega}\left(f_{i}, N\right) \mathbf{w}
\end{array}
$$

Given a $P_{F A}(=\alpha)$ to be achieved, the detection rule that maximizes $P_{D}$ is given by the Likelihood-Ratio Test:

$$
\frac{p_{\hat{\mathbf{S}}_{M T M}}\left(\hat{\mathbf{S}}_{M T M}^{\text {obs }} ; \mathcal{H}_{1}\right)}{p_{\hat{\mathbf{S}}_{M T M}}\left(\hat{\mathbf{S}}_{M T M}^{o b s} ; \mathcal{H}_{0}\right)} \underset{\mathcal{H}_{0}}{\gtrless} \xi
$$

where $\xi$ is appropriately chosen such that $P_{F A}=\alpha$.

\section{A. Optimal detection rule}

To examine the distribution of the random vector $\hat{\mathbf{S}}_{M T M}$ under both the hypotheses, it is useful to consider a vector representation of the MTM:

$$
\hat{S}_{M T M}^{k}\left(f_{i}\right)=\left|\sum_{n=0}^{N-1} x[n] v_{k}[n] e^{-j 2 \pi f_{i}}\right|^{2},
$$

where $\mathbf{v}_{\mathbf{k}}$ denotes the $k^{\text {th }}$ DPSW window and the frequency $f_{i}=i / N(i, k=0, \ldots, N-1)$. Then

$$
\hat{S}_{M T M}^{k}\left(f_{i}\right)=\left|\mathbf{b}_{k}^{H}\left(f_{i}\right) \mathbf{x}\right|^{2}=\mathbf{x}^{H} \mathbf{b}_{k}\left(f_{i}\right) \mathbf{b}_{k}^{H}\left(f_{i}\right) \mathbf{x}
$$

where the $N \times 1$ vector $\mathbf{b}_{k}$ is defined as the suitably frequency-shifted version of $\mathbf{v}_{k}$. The crude MTM estimate now becomes, when written compactly,

$$
\hat{S}_{M T M}\left(f_{i}\right)=\sum_{k=0}^{K-1} a_{k} \mathbf{x}^{H} \mathbf{b}_{k}\left(f_{i}\right) \mathbf{b}_{k}^{H}\left(f_{i}\right) \mathbf{x}=\mathbf{x}^{H} \boldsymbol{\Omega}\left(f_{i}\right) \mathbf{x}
$$

where the $N \times N$ frequency-dependent matrix $\Omega\left(f_{i}\right)$ is defined as

$$
\boldsymbol{\Omega}\left(f_{i}\right)=\sum_{k=0}^{K-1} a_{k} \mathbf{b}_{k}\left(f_{i}\right) \mathbf{b}_{k}^{H}\left(f_{i}\right)
$$

This quadratic form in the $\mathbf{x}$, under $\mathcal{H}_{0}(\mathbf{x}=\mathbf{w})$, can be shown to be a scaled chi-squared variable [6].

$$
\hat{S}_{M T M}\left(f_{i}\right) \stackrel{\mathcal{H}_{0}}{\approx} \frac{S_{0}\left(f_{i}\right)}{2 K} \cdot \chi_{2 K}^{2}
$$

where $S_{0}$ is the analytic spectrum of the received signal under $\mathcal{H}_{0}$. where $D\left(f_{i}\right)=\frac{S\left(f_{i}\right)}{2 K}$, a scaled version of the

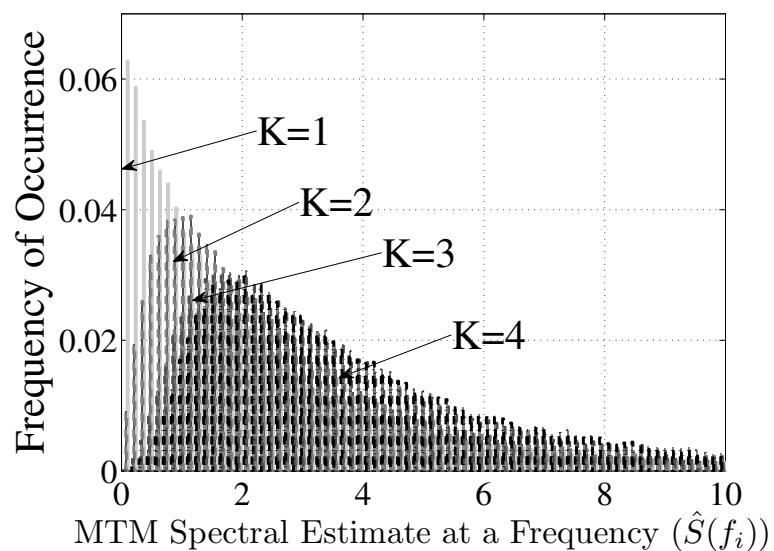

Figure 2: Histogram of the MTM Estimate at a Single Frequency

analytic spectrum. For frequencies $f_{i}, i=0,1,2, \ldots, N-1$,

$p_{\hat{S}_{M T M, 0}}\left(x\left(f_{i}\right)\right)=\frac{1}{2^{K} \Gamma_{K} D\left(f_{i}\right)}\left[\frac{x\left(f_{i}\right)}{D\left(f_{i}\right)}\right]^{K-1} e^{-\frac{1}{2 D\left(f_{i}\right)} x\left(f_{i}\right)}$

Under $\mathcal{H}_{1}$, the distribution is no longer exactly $\chi_{2 K}^{2}$ as $\hat{S}_{M T M}$ is a sum of squares of $2 K$ correlated nonzero mean normal variables. At non-zero frequencies, due to the low-leakage of the DPSW windows, the nonzero mean does not contribute significantly to the PSD estimate. As such we make the simplifying assumption that $\hat{S}_{M T M}$ is a scaled $\chi_{2 K}^{2}$ distributed variable (verified through simulations as shown in Figure 2). Also, the PSD estimates at different frequencies are correlated under both the hypotheses, making the joint PSD distribution difficult to analyze analytically. We therefore approximate the joint pdf as a product of individual densities assuming independence of estimates at different frequencies under both hypotheses. By choosing the leakage bandwidth $W$ appropriately $\left(W<\frac{1}{N}\right)$, the leakages can be approximated to be zero - justifying the aforementioned assumptions. The likelihood ratio under question can be written as

$$
\begin{aligned}
& L_{M T M}\left(\hat{\mathbf{S}}_{M T M}^{o b s}\right) \triangleq \frac{p_{\hat{\mathbf{S}}_{M T M}}\left(\hat{\mathbf{S}}_{M T M}^{o b s} ; \mathcal{H}_{1}\right)}{p_{\hat{\mathbf{S}}_{M T M}}\left(\hat{\mathbf{S}}_{M T M}^{o b s} ; \mathcal{H}_{0}\right)} \\
& =\frac{\prod_{i=0}^{N-1} p\left(\hat{S}_{M T M}\left(f_{i}\right)=\hat{S}_{M T M}^{o b s}\left(f_{i}\right) ; \mathcal{H}_{1}\right)}{\prod_{i=0}^{N-1} p\left(\hat{S}_{M T M}\left(f_{i}\right)=\hat{S}_{M T M}^{o b s}\left(f_{i}\right) ; \mathcal{H}_{0}\right)}
\end{aligned}
$$

(with a slight abuse of notation)

$$
=\frac{\prod_{i=0}^{N-1} \frac{1}{2^{K} \Gamma_{K} D_{1}\left(f_{i}\right)}\left[\frac{\hat{\mathbf{S}}_{M T M}^{o b s}}{D_{1}\left(f_{i}\right)}\right]^{K-1} e^{-\frac{\hat{S}_{M T M}^{o b s}\left(f_{i}\right)}{2 D_{0}\left(f_{i}\right)}}}{\prod_{i=0}^{N-1} \frac{1}{2^{K} \Gamma_{K} D_{0}\left(f_{i}\right)}\left[\frac{\hat{S}_{M T M}^{o b s}\left(f_{i}\right)}{D_{0}\left(f_{i}\right)}\right]^{K-1} e^{-\frac{\hat{S}_{M T M}^{o b s}\left(f_{i}\right)}{2 D_{0}\left(f_{i}\right)}}}
$$

On simplifying, the LRT now becomes

$$
=\prod_{i=0}^{N-1}\left(\frac{D_{0}^{K} e^{-\frac{\hat{S}_{M T M}^{o b s}\left(f_{i}\right)}{2 D_{1}\left(f_{i}\right)}}}{D_{1}^{K} e^{-\frac{\hat{S}_{M T M}^{o b s}\left(f_{i}\right)}{2 D_{0}\left(f_{i}\right)}}}\right) \underset{\mathcal{H}_{0}}{\stackrel{\mathcal{H}_{1}}{\gtrless}} \xi
$$




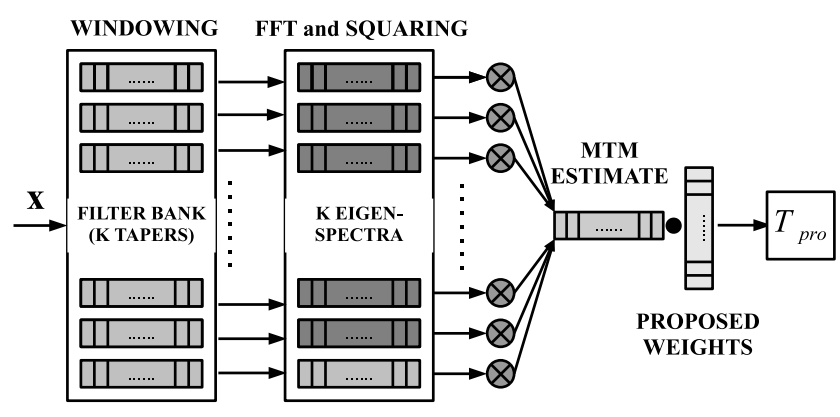

PERIODOGRAM LINEAR COMBINATION

Figure 3: Block Diagram of Proposed Detector

Taking logarithm on both the sides

$$
\begin{aligned}
\ln \left(L\left(\hat{\mathbf{S}}_{M T M}^{o b s}\right)\right) & =\sum_{i=0}^{N-1}\left(K \ln \left[\frac{D_{0}}{D_{1}}\right]\right. \\
+ & \left.\frac{1}{2}\left(\frac{1}{D_{0}\left(f_{i}\right)}-\frac{1}{D_{1}\left(f_{i}\right)}\right) \hat{S}_{M T M}^{o b s}\left(f_{i}\right)\right) \stackrel{\mathcal{H}_{1}}{\gtrless} \xi^{\prime}
\end{aligned}
$$

Subtracting out the constant terms on the LHS and substituting $D_{f_{i}}=\frac{S\left(f_{i}\right)}{2 K}$, after some simplification gives

$$
T_{\text {prop }}=\sum_{i=0}^{N-1}\left(\frac{1}{S_{0}\left(f_{i}\right)}-\frac{1}{S_{1}\left(f_{i}\right)}\right) \hat{S}_{M T M}^{o b s}\left(f_{i}\right) \underset{\mathcal{H}_{0}}{\stackrel{\mathcal{H}_{1}}{\gtrless}} \xi_{\text {prop }}
$$

Equation (25) is the proposed Neyman-Pearson optimal detection rule using MTM estimates. The test-statistic is a weighted combination of the MTM estimates. The weights are related to the difference in analytic spectra under the two hypotheses. In the stop-band which has only noise under $\mathcal{H}_{1}$, the analytic spectra are equal and the PSD estimates at those frequencies are ignored. On the other hand, at the tone frequency, the difference becomes large and is therefore weighted more, which makes intuitive sense. The weights, however, go as the inverse and not direct difference between spectra, as the higher valued spectral components are also affected by a higher variance in their PSD estimates. The inverse difference, in this regard, does not give as large a weighting to such high variance terms as a direct difference $\left(S_{1}\left(f_{i}\right)-S_{0}\left(f_{i}\right)\right)$ does and therefore the performance is not worsened due to increase in variance of statistic. The entire procedure of the proposed test is shown in Figure 3.

\section{B. Setting the threshold}

For the detector to achieve a predefined $P_{F A \text {,target }}(=$ $\beta$ ), the threshold needs to be set appropriately (in terms of $\beta$ ). Denoting by $\hat{\mathbf{S}}_{i}$ the vector of MTM estimates under $\mathcal{H}_{i}, P_{F A}$ can be expressed in terms of the threshold $\xi$ as:

$$
\begin{aligned}
& P_{F A}=P\left(T_{\text {prop }}>\xi ; \mathcal{H}_{0}\right) \\
& T_{\text {prop }}(\mathbf{x}) \stackrel{\mathcal{H}_{0}}{=} \sum_{i=0}^{N-1}\left(\frac{1}{S_{0}\left(f_{i}\right)}-\frac{1}{S_{1}\left(f_{i}\right)}\right) \hat{S}_{M T M}^{o b s}\left(f_{i}\right) \\
& =\sum_{i=0}^{N-1}\left(\frac{1}{S_{0}\left(f_{i}\right)}-\frac{1}{S_{1}\left(f_{i}\right)}\right) \sum_{k=0}^{K-1} a_{k} \mathbf{w}^{H} \mathbf{b}_{k}\left(f_{i}\right) \mathbf{b}_{k}^{H}\left(f_{i}\right) \mathbf{w}
\end{aligned}
$$

Looking at the expectations of the inner summands,

$$
E\left[\mathbf{w}^{H} \mathbf{b}_{k}\left(f_{i}\right) \mathbf{b}_{k}^{H}\left(f_{i}\right) \mathbf{w}\right]=\sigma_{w}^{2} \delta_{k, l}
$$

As both $\mathbf{b}$ vectors are at the same frequency, the corresponding frequency dependent factors cancel out to convert it to a dot-product of $\mathbf{v}$ vectors. These DPSW sequences as mentioned in Section III are form an orthogonal set, leading to (28). It can be seen that $\mathbf{b}_{k}^{H} \mathbf{w}$ and $\mathbf{b}_{k}^{H} \mathbf{w}$ $(k \neq l)$ are uncorrelated zero-mean normal variables. Then $\hat{S}_{M T M}^{o b s}\left(f_{i}\right)$ reduces to a weighted sum of squares of $K$ uncorrelated zero-mean Gaussian variables, distributed as:

$$
p_{\hat{S}_{M T M}^{o b s}}(x)=\left[\prod_{i=1}^{n} \lambda_{i}\right] \sum_{j=1}^{K} \frac{e^{-\lambda_{j} x}}{\prod_{\substack{k \neq j \\ k=1}}^{K} \lambda_{k}-\lambda_{j}}, \quad x>0 .
$$

where $\lambda_{j}=\left(\left|a_{j}\right|^{2} \sigma_{w}^{2}\right)^{-1}$. $\hat{S}_{M T M}^{o b s}\left(f_{i}\right)$ is a sum of $K$ independent exponential random variables with different means. As we consider $K$ values that are sufficiently large, using extensions of the CLT [6], we can approximate the resultant, and thereby the test-statistic, as a Gaussian variable. The mean $\left(\mu_{0}\right)$ and variance $\left(\sigma_{0}^{2}\right)$ are given as:

$\mu_{0}=\sum_{i=0}^{N-1}\left(\frac{1}{S_{0}\left(f_{i}\right)}-\frac{1}{S_{1}\left(f_{i}\right)}\right) S_{0}\left(f_{i}\right)$

$\sigma_{0}^{2}=\sum_{i=0}^{N-1} \sigma_{w}^{4} \sum_{k=0}^{K-1} a_{k}^{2}+\sum_{i_{1,2}=0}^{N-1} \sum_{k_{1,2}=0}^{K-1} a_{k_{1}} a_{k_{2}}\left|\mathbf{b}_{k_{2}}^{2}\left(f_{i_{2}}\right) \mathbf{b}_{k_{1}}\left(f_{i_{1}}\right)\right|^{2}$

We omit the derivations due to lack of space. Then,

$$
P_{F A}=P\left(T>\xi ; \mathcal{H}_{0}\right)=Q\left(\frac{\xi-\mu_{0}}{\sigma_{0}}\right)
$$

Inverting the above relation, we obtain

$$
\xi=\mu_{0}+\sigma_{0} \cdot Q^{-1}\left(P_{F A}\right)
$$

In our simulations, we use data blocks of length $N=128$ or 256 - which correspond to a small fraction of the typical coherence times associated with the high-frequency HDTV channels and hence forsake time-diversity gains. One possible solution is to improve spectral estimates by averaging out over multiple coherence time windows (without necessarily changing the block length). While improving diversity gain, this does not severely affect the threshold analysis. Under $\mathcal{H}_{0}$, the new test statistic is still Gaussian with the same mean and variance decreased by a factor of $N_{a v}$ (number of time windows) due to noise being uncorrelated across time windows.

$$
\mu_{0, a v g}=\mu_{0}, \quad \sigma_{0, a v g}^{2}=\frac{\sigma_{0}^{2}}{N_{a v}}
$$

The detector of Wang et al assumes the presence of

\begin{tabular}{|c|c|c|c|}
\hline Detector & $\mathcal{O}(N)$ & Detector & $\mathcal{O}(N)$ \\
\hline Energy Detector & $N$ & Proposed Rule & $N \log N$ \\
Wang, Chiang & $N \log N$ & Cyclostationary & $N^{2}$ \\
\hline
\end{tabular}

Table I: Comparison of Computational Complexities 
a pilot signal, thereby also leading to simplification of analysis. The Chiang et al rule, besides being sub-optimal, is difficult to analyze [11]. The proposed detector, while being optimal, permits the threshold to be set easily. We emphasize that the proposed detector is of relatively low complexity when compared to some of the commonly used detectors. As the proposed detector has $K$ windowing operations, FFTs and weighted additions, its complexity lies in $\mathcal{O}(N \log N)$. A comparison with some other detectors is shown in Table I. The proposed detector can be expressed as $F F T$ based spectral estimation followed by filtering operation (Figure 3), thereby admitting an easy implementation as well.

\section{Numerical RESUlts}

To verify the performance of our proposed detector, we simulate the detection rule in MATLAB with HDTV data. We generate HDTV data of sufficient length and apply the MTM. $K$ is varied between 2 to 16 to find the optimal number empirically while using a suitable block size of 128 or $256(N)$. We implement the three different rules mentioned in this chapter - the Chiang et al test, the Wang et al "tone-detection" algorithm, our proposed detection rule and also energy detection using sufficiently large number of iterations (around $10^{4}$ ), and simultaneously over multiple fading coefficients for the quasi-statistic to get the average performance over fading. Note that we iterate and not average the test statistic over multiple coherence windows. We consider SNR in the range of 0 to $-40 d B$, which corresponds to actual range of operation. The IEEE 802.22 guidelines mention that detection should be possible with low $P_{F A}$ (around 0.1 - 0.2) and $P_{M D}$ (around $10^{-2}$ ) at signal power as low as $-125 d B$. This is, obviously, the upper limit on the least SNR required for operation. We use small data windows to ease our simulations and as such do not try to reach the benchmarks mentioned. We compare the various schemes under consideration and note that the required performance can be obtained by suitably increasing the window size.

The performance ( $P_{M D}$ vs. SNR) of each scheme is plotted while varying $P_{F A}$ over over a large range (plotted for two values of $P_{F A}$ in Figures 4,5). We observe that for almost all values of $P_{F A}$ and SNR, the proposed detection rule clearly outperforms the other methods. Note that the value of drastic performance decay (in $P_{M D}$ ) occurs at a much lower value of SNR than the other two methods.

\section{CONCLUSION}

In this work the problem of spectral estimation in the context of cognitive radios designed to operate in HDTV whitespaces is considered. We put forth the idea of spectral estimation followed by optimal detection. Choosing Thomson's multi taper method for spectral estimation, we frame the problem of finding the optimal detector following this. The optimal detection rule under suitable approximations, as well as the appropriate threshold for it, are derived. The proposed detector's performance is evaluated through simulations using actual HDTV data. Lastly, the solution is compared to previous attempts in this direction and the performance improvements, reduced computational complexity among other benefits are highlighted. The essential

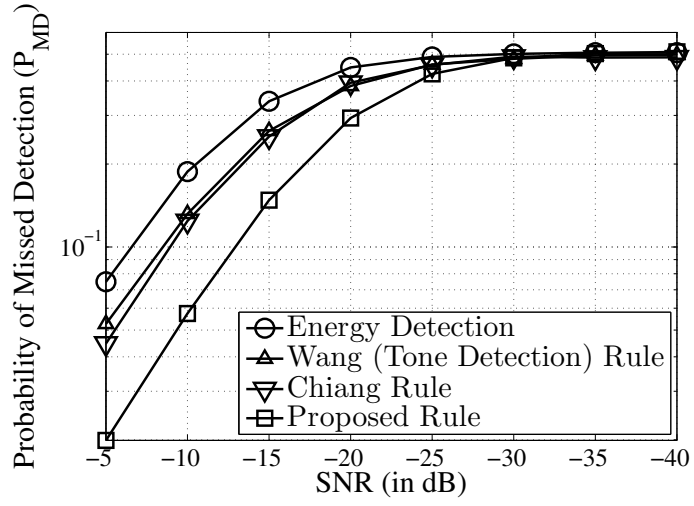

Figure 4: Performance of Proposed Scheme $\left(P_{F A}=0.5\right)$

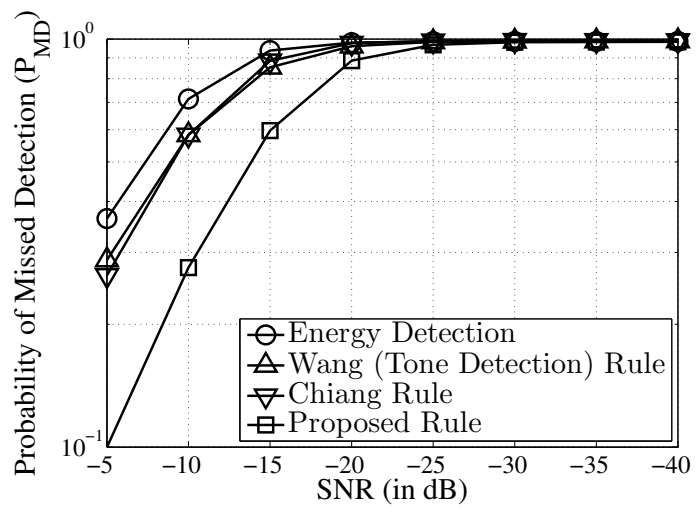

Figure 5: Performance of Proposed Scheme $\left(P_{F A}=0.01\right)$

idea of optimal detection following estimation could be extended to other system models as well.

\section{REFERENCES}

[1] J. Mitola, "The software radio architecture," Communications Magazine, IEEE, vol. 33, no. 5, pp. 26-38, 1995.

[2] S. Force, "Spectrum policy task force report," Federal Communications Commission ET Docket 02, vol. 135, 2002.

[3] S. Haykin, D. Thomson, and J. Reed, "Spectrum sensing for cognitive radio," Proceedings of the IEEE, vol. 97, no. 5, pp. 849877, 2009.

[4] C. Cordeiro, K. Challapali, D. Birru, S. Shankar et al., "Ieee 802.22: An introduction to the first wireless standard based on cognitive radios," Journal of communications, vol. 1, no. 1, pp. 38-47, 2006.

[5] W. Bretl, W. Meintel, G. Sgrignoli, X. Wang, S. Weiss, and K. Salehian, "Atsc rf, modulation, and transmission," Proceedings of the IEEE, vol. 94, no. 1, pp. 44-59, 2006.

[6] D. Percival and A. Walden, Spectral analysis for physical applications: multitaper and conventional univariate techniques. Cambridge Univ Pr, 1993.

[7] C. Jarque and A. Bera, "Efficient tests for normality, homoscedasticity and serial independence of regression residuals," Economics Letters, vol. 6, no. 3, pp. 255-259, 1980.

[8] D. Thomson, "Spectrum estimation and harmonic analysis," Proceedings of the IEEE, vol. 70, no. 9, pp. 1055-1096, 1982.

[9] A. Drosopoulos and S. Haykin, "Adaptive radar parameter estimation with thomson's multiple window method," Adaptive Radar Detection and Estimation. John Wiley, New York, 1992.

[10] D. Slepian, "Prolate spheroidal wave functions, fourier analysis, and uncertainty. v-the discrete case," AT T Technical Journal, vol. 57, pp. 1371-1430, 1978

[11] T. Chiang, J. Lin, and H. Ma, "Optimal detector for multitaper spectrum estimator in cognitive radios," in Global Telecommunications Conference, 2009, pp. 1-6.

[12] J. Wang and Q. Zhang, "A multitaper spectrum based detector for cognitive radio," in Wireless Communications and Networking Conference, 2009, pp. 1-5. 